\title{
Three color Ramsey numbers for graphs with at most 4 vertices
}

\author{
Luis Boza \\ University of Sevilla \\ Department of Applied Mathematics I \\ Reina Mercedes 2, 41012-Seville, Spain \\ boza@us.es
}

\author{
Janusz Dybizbański Tomasz Dzido \\ University of Gdańsk \\ Institute of Informatics \\ Wita Stwosza 57, 80-952 Gdańsk, Poland \\ jdybiz@inf.ug.edu.pl, tdz@inf.ug.edu.pl
}

Submitted: Mar 23, 2012; Accepted: Dec 8, 2012; Published: Dec 31, 2012

Mathematics Subject Classifications: 05C55, 05D10

\begin{abstract}
For given graphs $H_{1}, H_{2}, H_{3}$, the 3-color Ramsey number $R\left(H_{1}, H_{2}, H_{3}\right)$ is the smallest integer $n$ such that if we arbitrarily color the edges of the complete graph of order $n$ with 3 colors, then it always contains a monochromatic copy of $H_{i}$ colored with $i$, for some $1 \leqslant i \leqslant 3$.

We study the bounds on 3-color Ramsey numbers $R\left(H_{1}, H_{2}, H_{3}\right)$, where $H_{i}$ is an isolate-free graph different from $K_{2}$ with at most four vertices, establishing that $R\left(P_{4}, C_{4}, K_{4}\right)=14, R\left(C_{4}, K_{3}, K_{4}-e\right)=17, R\left(C_{4}, K_{3}+e, K_{4}-e\right)=17, R\left(C_{4}, K_{4}-\right.$ $\left.e, K_{4}-e\right)=19,28 \leqslant R\left(C_{4}, K_{4}-e, K_{4}\right) \leqslant 36, R\left(K_{3}, K_{4}-e, K_{4}\right) \leqslant 41, R\left(K_{4}-e, K_{4}-\right.$ $\left.e, K_{4}\right) \leqslant 59$ and $R\left(K_{4}-e, K_{4}, K_{4}\right) \leqslant 113$. Also, we prove that $R\left(K_{3}+e, K_{4}-e, K_{4}-\right.$ $e)=R\left(K_{3}, K_{4}-e, K_{4}-e\right), R\left(C_{4}, K_{3}+e, K_{4}\right) \leqslant \max \left\{R\left(C_{4}, K_{3}, K_{4}\right), 29\right\} \leqslant 32$, $R\left(K_{3}+e, K_{4}-e, K_{4}\right) \leqslant \max \left\{R\left(K_{3}, K_{4}-e, K_{4}\right), 33\right\} \leqslant 41$ and $R\left(K_{3}+e, K_{4}, K_{4}\right) \leqslant$ $\max \left\{R\left(K_{3}, K_{4}, K_{4}\right), 2 R\left(K_{3}, K_{3}, K_{4}\right)+2\right\} \leqslant 79$.

This paper is an extension of the article by Arste, Klamroth, Mengersen [Utilitas Mathematica, 1996].
\end{abstract}

\section{Introduction}

In this paper all graphs considered are undirected, finite and contain neither loops nor multiple edges. Let $G$ be such a graph. The vertex set of $G$ is denoted by $V(G)$, the edge set of $G$ by $E(G)$, and the number of edges in $G$ by $e(G)$. The degree of the vertex $v$ and the number of the edge incident to $v$ colored with color $i$ are denoted with $d(v)$ and $d_{i}(v)$, respectively. By $\delta_{i}(G)$ and $\Delta_{i}(G)$ we denote the minimum and the maximum degree of vertices in $G$ that are colored with color $i$, respectively. The open neighborhood of vertex $v$ in color $i$ in graph $G$ is $N_{i}(v)=\{u \in V(G) \mid\{u, v\} \in$ $E(G)$ and $\{u, v\}$ is colored with color $i\}$. Define $G[S]$ to be a subgraph of $G$ induced by 
a set of vertices $S \subseteq V(G)$ and $G^{i}$ to be a graph induced by the edges of $G$ colored with color $i$. Let $P_{n}$ (resp. $C_{n}$ ) be the path (resp. cycle) on $n$ vertices. The cardinality of a set $A$ is denoted by $|A|$.

For given graphs $G_{1}, G_{2}, \ldots, G_{k}, k \geqslant 2$, the multicolor Ramsey number $R\left(G_{1}, G_{2}, \ldots\right.$, $G_{k}$ ) is the smallest integer $n$ such that if we arbitrarily color the edges of the complete graph of order $n$ with $k$ colors, then it always contains a monochromatic copy of $G_{i}$ colored with the color $i$, for some $1 \leqslant i \leqslant k$. A coloring of the edges of $n$-vertex complete graph with $m$ colors is called a $\left(G_{1}, G_{2}, \ldots, G_{m} ; n\right)$-coloring, if it does not contain a subgraph isomorphic to $G_{i}$ colored with the color $i$, for each $i$. The set of all non-isomorphic $\left(G_{1}, \ldots, G_{m} ; n\right)$-colorings is denoted by $\left(G_{1}, \ldots, G_{m} ; n\right)$. We refer to consecutive colors corresponding to the parameters of colorings as red, blue and green.

In this paper we consider isolate-free graphs different from $K_{2}$ with at most four vertices, improving some results from the article by Arste, Klamroth, Mengersen [1] from 1996 and [9].

Note that $R\left(H_{1}, H_{2}, H_{3}\right)=R\left(H_{\sigma(1)}, H_{\sigma(2)}, H_{\sigma(3)}\right)$ for every permutation $\sigma$ of $\{1,2,3\}$. The case $K_{2}$ is omitted here, because $R\left(K_{2}, H_{2}, H_{3}\right)=R\left(H_{2}, H_{3}\right)$ and these numbers were already determined in $[6,7,12]$.

We use the following two formulas, which are very well known:

$R\left(H_{1}^{\prime}, H_{2}^{\prime}, H_{3}^{\prime}\right) \geqslant R\left(H_{1}, H_{2}, H_{3}\right)$, if $H_{i}$ is a subgraph of $H_{i}^{\prime}$.

$R\left(H_{1}, H_{2}, H_{3}\right) \leqslant R\left(H_{1}-v_{1}, H_{2}, H_{3}\right)+R\left(H_{1}, H_{2}-v_{2}, H_{3}\right)+R\left(H_{1}, H_{2}, H_{3}-v_{3}\right)-1$, if $v_{i} \in V\left(H_{i}\right)$, with strict inequality when the right-hand-side and at least one of its terms are even.

\section{Results}

The values $R\left(H_{1}, H_{2}, H_{3}\right)$ are known if:

- some $H_{i}$ is $P_{3}, 2 K_{2}, P_{4}$ or $K_{1,3}$, except $R\left(P_{4}, C_{4}, K_{4}\right)[1,4,5,10,15,17]$,

- some $H_{i}$ is $C_{4}, K_{3}, K_{3}+e$ or $K_{4}-e$ and the other are $C_{4}, K_{3}$ or $K_{3}+e$, except $R\left(K_{3}, C_{4}, K_{4}-e\right)$ and $R\left(K_{3}+e, C_{4}, K_{4}-e\right)[1,3,9,11,12,26,27]$.

Also, bounds of other values are known [1, 9, 10, 16, 18, 22, 23, 27, 28].

In this paper we improve the lower bound $R\left(C_{4}, K_{4}-e, K_{4}\right) \geqslant 27$ obtained from (1). Also, we improve the upper bounds $R\left(C_{4}, K_{3}, K_{4}-e\right) \leqslant 25, R\left(C_{4}, K_{3}+e, K_{4}-e\right) \leqslant 25$, $R\left(C_{4}, K_{3}+e, K_{4}\right) \leqslant 43, R\left(C_{4}, K_{4}-e, K_{4}\right) \leqslant 54, R\left(K_{3}, K_{4}-e, K_{4}\right) \leqslant 44, R\left(K_{3}+e, K_{4}-\right.$ $\left.e, K_{4}\right) \leqslant 44, R\left(K_{4}-e, K_{4}-e, K_{4}\right) \leqslant 60$ and $R\left(K_{4}-e, K_{4}, K_{4}\right) \leqslant 121$, obtained from (2), as well as $R\left(P_{4}, C_{4}, K_{4}\right) \leqslant 15[1]$ and $R\left(C_{4}, K_{4}-e, K_{4}-e\right) \leqslant 22[9]$.

We prove that $R\left(P_{4}, C_{4}, K_{4}\right)=14, R\left(C_{4}, K_{3}, K_{4}-e\right)=17, R\left(C_{4}, K_{3}+e, K_{4}-e\right)=17$, $R\left(C_{4}, K_{4}-e, K_{4}-e\right)=19,28 \leqslant R\left(C_{4}, K_{4}-e, K_{4}\right) \leqslant 36, R\left(K_{3}, K_{4}-e, K_{4}\right) \leqslant 41$, $R\left(K_{4}-e, K_{4}-e, K_{4}\right) \leqslant 59$ and $R\left(K_{4}-e, K_{4}, K_{4}\right) \leqslant 113$. 
For some classes of $G$ and $H$, it was known that $R\left(K_{3}+e, G, H\right)=R\left(K_{3}, G, H\right)$, for instance $R\left(K_{3}+e, K_{3}+e, K_{4}\right)=R\left(K_{3}, K_{3}+e, K_{4}\right)=R\left(K_{3}, K_{3}, K_{4}\right)$ [1] and $R\left(K_{3}+\right.$ $\left.e, K_{3}+e, K_{4}-e\right)=R\left(K_{3}, K_{3}+e, K_{4}-e\right)=R\left(K_{3}, K_{3}, K_{4}-e\right)$ [27]. We prove that $R\left(K_{3}+e, K_{4}-e, K_{4}-e\right)=R\left(K_{3}, K_{4}-e, K_{4}-e\right)$. Also, we prove that $R\left(C_{4}, K_{3}+e, K_{4}\right) \leqslant$ $\max \left\{R\left(C_{4}, K_{3}, K_{4}\right), 29\right\} \leqslant 32, R\left(K_{3}+e, K_{4}-e, K_{4}\right) \leqslant \max \left\{R\left(K_{3}, K_{4}-e, K_{4}\right), 33\right\} \leqslant 41$ and $R\left(K_{3}+e, K_{4}, K_{4}\right) \leqslant \max \left\{R\left(K_{3}, K_{4}, K_{4}\right), 2 R\left(K_{3}, K_{3}, K_{4}\right)+2\right\} \leqslant 79$.

Now, we give the values and bounds of $R\left(H_{1}, H_{2}, H_{3}\right)$ in the following tables. Although the first two complete tables and parts of the remaining tables are shown in [1], for the sake of completeness, we repeat them below. In these tables, the lower bounds obtained from (1) are marked with a "*". Also, we will mark with " $\nmid$ " the upper bounds obtained using (2). We use bold style to denote the new values or bounds presented in this paper.

\begin{tabular}{|c|c|c|c|c|c|c|c|c|c|}
\hline$P_{3}$ & $5[5]$ & & & & & & & & \\
\hline $2 K_{2}$ & $4_{[1]}$ & $5_{[1]}$ & & & & & & & \\
\hline$P_{4}$ & $5_{[1]}$ & $5[1]$ & $5[1]$ & & & & & & \\
\hline$K_{1,3}$ & $5[5]$ & $6[1]$ & $7_{[1]}$ & $7_{[5]}$ & & & & & \\
\hline$C_{4}$ & $6_{[1]}$ & $6_{[1]}$ & $7_{[1]}$ & $7_{[17]}$ & $8_{[1]}$ & & & & \\
\hline$K_{3}$ & 5 [15] & $6_{[1]}$ & $7_{[1]}$ & $9_{[15]}$ & $8_{[1]}$ & $11_{[4]}$ & & & \\
\hline$K_{3}+e$ & $5[1]$ & $6_{[1]}$ & $7_{[1]}$ & $9_{[1]}$ & $8[1]$ & $11_{[1]}$ & $11_{[1]}$ & & \\
\hline$K_{4}-e$ & $7_{[1]}$ & $6_{[1]}$ & $7_{[1]}$ & $9_{[1]}$ & $9_{[1]}$ & $11_{[1]}$ & $11_{[1]}$ & $11_{[10]}$ & \\
\hline$K_{4}$ & $7_{[15]}$ & $8[1]$ & $10_{[1]}$ & $13_{[15]}$ & $13_{[1]}$ & $17_{[4]}$ & $17_{[1]}$ & $17_{[1]}$ & $35_{[4]}$ \\
\hline$P_{3}$ & $P_{3}$ & $2 K_{2}$ & $P_{4}$ & $K_{1,3}$ & $C_{4}$ & $K_{3}$ & $K_{3}+e$ & $K_{4}-e$ & $K_{4}$ \\
\hline
\end{tabular}

Table 1: Values of $R\left(P_{3}, H_{1}, H_{2}\right)$.

\begin{tabular}{|c|c|c|c|c|c|c|c|c|}
\hline $2 K_{2}$ & $6_{[17]}$ & & & & & & & \\
\hline$P_{4}$ & $6_{[17]}$ & $6_{[17]}$ & & & & & & \\
\hline$K_{1,3}$ & $6_{[17]}$ & $6_{[17]}$ & $7_{[17]}$ & & & & & \\
\hline$C_{4}$ & $6_{[17]}$ & $6_{[17]}$ & $7_{[17]}$ & $7_{[17]}$ & & & & \\
\hline$K_{3}$ & $7_{[17]}$ & $8_{[17]}$ & $8_{[17]}$ & $8_{[15]}$ & $8_{[17]}$ & & & \\
\hline$K_{3}+e$ & $7_{[17]}$ & $8_{[17]}$ & $8_{[17]}$ & $8_{[17]}$ & $8_{[17]}$ & $8_{[17]}$ & & \\
\hline$K_{4}-e$ & $7_{[17]}$ & $8_{[17]}$ & $8_{[17]}$ & $9_{[17]}$ & $8_{[17]}$ & $8_{[17]}$ & $11_{[17]}$ & \\
\hline$K_{4}$ & $8_{[17]}$ & $11_{[17]}$ & $11_{[17]}$ & $11_{[17]}$ & $11_{[17]}$ & $11_{[17]}$ & $13_{[17]}$ & $20_{[17]}$ \\
\hline $2 K_{2}$ & $2 K_{2}$ & $P_{4}$ & $K_{1,3}$ & $C_{4}$ & $K_{3}$ & $K_{3}+e$ & $K_{4}-e$ & $K_{4}$ \\
\hline
\end{tabular}

Table 2: Values of $R\left(2 K_{2}, H_{1}, H_{2}\right)$. 


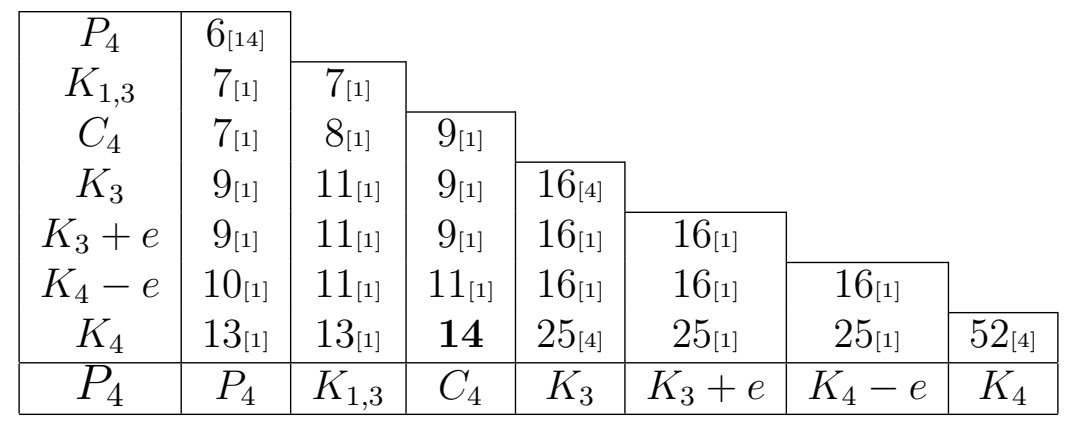

Table 3: Values of $R\left(P_{4}, H_{1}, H_{2}\right)$.

\begin{tabular}{|c|c|c|c|c|c|c|}
\hline \multirow{3}{*}{$\begin{array}{c}K_{1,3} \\
C_{4} \\
K_{2}\end{array}$} & \multirow{2}{*}{$\begin{array}{l}8_{[5]} \\
8_{[1]}\end{array}$} & \multirow[b]{2}{*}{$9_{[1]}$} & \multirow[b]{3}{*}{$16[4]$} & & & \\
\hline & & & & & & \\
\hline & $11_{[15]}$ & $11_{[1]}$ & & & & \\
\hline$K_{3}+e$ & $11_{[1]}$ & $11_{[1]}$ & $16_{[1]}$ & $16_{[1]}$ & & \\
\hline$K_{4}-e$ & $11_{[1]}$ & $11_{[1]}$ & $16_{[1]}$ & $16_{[1]}$ & $16_{[1]}$ & \\
\hline$K_{4}$ & $16_{[15]}$ & $16_{[17]}$ & $25_{[4]}$ & $25[1]$ & $25_{[1]}$ & $52_{[4]}$ \\
\hline$K_{1,3}$ & $K_{1,3}$ & $C_{4}$ & $K_{3}$ & $K_{3}+e$ & $K_{4}-e$ & $K_{4}$ \\
\hline
\end{tabular}

Table 4: Values of $R\left(K_{1,3}, H_{1}, H_{2}\right)$.

\begin{tabular}{|c|c|c|c|c|c|}
\hline \multirow{5}{*}{$\begin{array}{c}C_{4} \\
K_{3} \\
K_{3}+e \\
K_{4}-e \\
K_{4}\end{array}$} & \multirow{2}{*}{$\begin{array}{c}11_{[3]} \\
12_{[26]}\end{array}$} & \multirow[b]{2}{*}{$17_{[11]}$} & \multirow[b]{3}{*}{$17_{[1]}$} & \multirow{5}{*}{$\begin{array}{c}19 \\
28-36\end{array}$} & \\
\hline & & & & & \\
\hline & $12_{[1]}$ & $17_{[1]}$ & & & \\
\hline & 16 & 17 & 17 & & \\
\hline & $20_{[9]-}-22_{[28]}$ & $27_{[9]-32_{[28]}}$ & $27 *-32$ & & $52_{*}-72_{[28]}$ \\
\hline$C_{4}$ & $C_{4}$ & $K_{3}$ & $K_{3}+e$ & $K_{4}-e$ & $K_{4}$ \\
\hline
\end{tabular}

Table 5: Values and bounds of $R\left(C_{4}, H_{1}, H_{2}\right)$.

\begin{tabular}{|c|c|c|c|c|}
\hline \multirow{4}{*}{$\begin{array}{c}K_{3} \\
K_{3}+e \\
K_{4}-e \\
K_{4}\end{array}$} & \multirow{4}{*}{$\begin{array}{c}17_{[12]} \\
17_{[1]} \\
17_{[27]} \\
30_{[16]-31_{[23]}}\end{array}$} & \multirow{4}{*}{$\begin{array}{c}17_{[1]} \\
17_{[27]} \\
R\left(K_{3}, K_{3}, K_{4}\right)_{[1]}\end{array}$} & \multirow{4}{*}{$\begin{array}{c}21_{[27]-27_{[27]}} \\
30_{*}-\mathbf{4 1} \\
\end{array}$} & \\
\hline & & & & \\
\hline & & & & \\
\hline & & & & $55_{[18]}-79_{\dagger}$ \\
\hline$K_{3}$ & $K_{3}$ & $K_{3}+e$ & $K_{4}-e$ & $K_{4}$ \\
\hline
\end{tabular}

Table 6: Values and bounds of $R\left(K_{3}, H_{1}, H_{2}\right)$.

\section{Proofs}

$3.1 R\left(P_{4}, H_{1}, H_{2}\right)$

In [1] it is claimed that $14 \leqslant R\left(P_{4}, C_{4}, K_{4}\right) \leqslant 15$, but no $\left(P_{4}, C_{4}, K_{4} ; 13\right)$-coloring is showed. A $\left(P_{4}, C_{4}, K_{4} ; 13\right)$-coloring can be found in the Appendix. In this subsection we 


\begin{tabular}{|c|c|c|c|}
\hline$K_{3}+e$ & $17_{[29]}$ & \multicolumn{2}{|c}{} \\
$K_{4}-e$ & $17_{[27]}$ & $\boldsymbol{R}\left(\boldsymbol{K}_{\mathbf{3}}, \boldsymbol{K}_{\mathbf{4}}-\boldsymbol{e}, \boldsymbol{K}_{\mathbf{4}}-\boldsymbol{e}\right)$ & \\
\cline { 4 - 4 }$K_{4}$ & $R\left(K_{3}, K_{3}, K_{4}\right)[1]$ & $30_{*}-\mathbf{4 1}$ & $55_{*-79}$ \\
\hline$K_{3}+e$ & $K_{3}+e$ & $K_{4}-e$ & $K_{4}$ \\
\hline
\end{tabular}

Table 7: Values and bounds of $R\left(K_{3}+e, H_{1}, H_{2}\right)$.

\begin{tabular}{|c|c|c|}
\hline$K_{4}-e$ & $28_{[10]-30_{[22]}}$ & \\
$K_{4}$ & $33_{[27]-59}$ & $55_{*} \mathbf{1 1 3}$ \\
\hline$K_{4}-e$ & $K_{4}-e$ & $K_{4}$ \\
\hline
\end{tabular}

Table 8: Bounds on $R\left(K_{4}-e, H_{1}, H_{2}\right)$.

\begin{tabular}{|c|c|}
\hline$K_{4}$ & $128_{[13]-236}$ \\
\hline$K_{4}$ & $K_{4}$ \\
\hline
\end{tabular}

Table 9: Bounds on $R\left(K_{4}, K_{4}, K_{4}\right)$.

will prove that $R\left(P_{4}, C_{4}, K_{4}\right)=14$.

Let $F$ be a $P_{4}$-free graph and let $S(F)$ be the set of $\left(P_{4}, C_{4}, K_{4} ;|V(F)|\right)$-colorings of $G$ such that $G^{1}=F$.

We note that $R\left(P_{4}, C_{4}, K_{4}\right)=14$ if and only if for any $P_{4}$-free graph $F$ of order 14 $S(F)$ is empty.

Let $H$ and $H^{\prime}$ be two $P_{4}$-free graphs. It is easy to obtain $S\left(H^{\prime}\right)$ from $S(H)$ if one of the following two algorithms is applied:

Case a) $H$ is an induced subgraph of $H^{\prime}$ such that $\left|V\left(H^{\prime}\right)\right|=|V(H)|+1$.

Algorithm 1.

Input: coloring $G \in S(H)$.

Output: set of all one-vertex extensions of $G$ which are in $S\left(H^{\prime}\right)$.

For instance, if we consider the coloring $G$ belonging to $S\left(K_{2} \cup K_{1}\right)$ with 3 vertices, such that the edge $\{1,2\}$ is red and both $\{1,3\}$ and $\{2,3\}$ are blue, then we obtain two colorings of four vertices belonging to $S\left(2 K_{2}\right)$, adding a new vertex, assigning red color to $\{3,4\}$, green color to $\{1,4\}$ and blue or green color to $\{2,4\}$. The coloring such that $\{1,4\}$ and $\{2,4\}$ are blue, is not considered because it contains a blue $C_{4}$. The coloring such that $\{1,4\}$ is blue and $\{2,4\}$ is green, is not considered because it is isomorphic to coloring in which $\{1,4\}$ is green and $\{2,4\}$ is blue.

Case b) $H^{\prime}$ is a subgraph of $H$ such that $\left|V\left(H^{\prime}\right)\right|=|V(H)|$.

Algorithm 2.

Input: coloring $G \in S(H)$.

Output: set of all colorings of $S\left(H^{\prime}\right)$ obtained assigning blue or green color to some edges of $G$.

For instance, if we consider the coloring $G$ belonging to $S\left(2 K_{2} \cup K_{1}\right)$ with 5 vertices, such that the edges $\{1,2\}$ and $\{3,4\}$ are red, $\{1,5\}$ is blue and the remaining seven 
edges are green, then we obtain three colorings of five vertices belonging to $S\left(K_{2} \cup 3 K_{1}\right)$, assigning blue color to $\{3,4\}$ or assigning blue or green color to $\{1,2\}$. The coloring obtained assigning green color to $\{3,4\}$ is not considered because it contains a green $K_{4}$.

The lists of graphs generated in order to prove that $S(F)=\emptyset$ for any $P_{4}$-free graph $F$ of order 14 are not very large, thus, it is not necessary to utilize the program nauty to eliminate graph isomorphisms $[20,21]$. To check isomorphisms of graphs, we use the IsomormicQ command of the Combinatorica package of the Mathematica 8.0 program [19].

We use the following result:

Lemma 3. Let $F_{1}, F_{2}$ and $F_{3}$ be three graphs. If $S\left(F_{1}\right)=\emptyset, F_{3}$ is a subgraph of $F_{1}$ with $V\left(F_{3}\right)=V\left(F_{1}\right)$ and $F_{3}$ is an induced subgraph of $F_{2}$, then $S\left(F_{2}\right)=\emptyset$.

Proof. Applying Algorithm 2 we obtain $S\left(F_{3}\right)$ from $S\left(F_{1}\right)$, thus $S\left(F_{3}\right)=\emptyset$, and applying Algorithm 1 we obtain $S\left(F_{2}\right)$ from $S\left(F_{3}\right)$, hence $S\left(F_{2}\right)=\emptyset$.

In order to prove that for any $P_{4}$-free graph $F$ of order $14, S(F)=\emptyset$, without loss of generality we can assume that the components of $F$ are $K_{2}, K_{3}$ or $K_{1, n}$, with $n \geqslant 3$, because if $K_{1}$ or $P_{3}$ are components of $F$, there exists a graph $F_{0}$ or order 14 such that $F$ is a subgraph of $F_{0}$ and $K_{1}$ and $P_{3}$ are not components of $F_{0}$. Thus, if $S\left(F_{0}\right)=\emptyset$ then, applying Algorithm 2, we have that $S(F)=\emptyset$.

Since $R\left(C_{4}, K_{4}\right)=10$ [7], $F_{0}$ has no independent set of order 10 and, since $R\left(P_{3}, C_{4}\right.$, $\left.K_{4}\right)=13, F_{0}$ has at least two components different of $K_{2}$. Thus, it is easy to check that $F_{0} \in\left\{4 K_{3} \cup K_{2}, 3 K_{3} \cup K_{1,4}, 2 K_{3} \cup K_{1,7}, 2 K_{3} \cup K_{1,5} \cup K_{2}, 2 K_{3} \cup 2 K_{1,3}, 2 K_{3} \cup K_{1,3} \cup\right.$ $\left.2 K_{2}, 2 K_{3} \cup 4 K_{2}, K_{3} \cup K_{1,6} \cup 2 K_{2}, K_{3} \cup K_{1,4} \cup K_{1,3} \cup K_{2}, K_{3} \cup K_{1,4} \cup 3 K_{2}, 2 K_{1,3} \cup 3 K_{2}\right\}$.

From Lemma 3, we obtain:

Corollary 4. If $S\left(K_{3} \cup 2 K_{2} \cup 5 K_{1}\right)=\emptyset$ then $S\left(2 K_{3} \cup K_{1,7}\right), S\left(2 K_{3} \cup K_{1,5} \cup K_{2}\right), S\left(K_{3} \cup\right.$ $\left.K_{1,6} \cup 2 K_{2}\right), S\left(K_{3} \cup K_{1,4} \cup K_{1,3} \cup K_{2}\right)$ and $S\left(2 K_{1,3} \cup 3 K_{2}\right)=\emptyset$.

Proof. Let $F_{1}=K_{3} \cup 2 K_{2} \cup 5 K_{1}$. Considering $F_{3}=K_{3} \cup 2 K_{2} \cup 5 K_{1}$ we have that $S\left(2 K_{3} \cup K_{1,5} \cup K_{2}\right), S\left(K_{3} \cup K_{1,6} \cup 2 K_{2}\right)=\emptyset$. Considering $F_{3}=K_{3} \cup K_{2} \cup 7 K_{1}$ we obtain that $S\left(2 K_{3} \cup K_{1,7}\right), S\left(K_{3} \cup K_{1,4} \cup K_{1,3} \cup K_{2}\right)=\emptyset$. Finally, considering $F_{3}=3 K_{2} \cup 6 K_{1}$ we obtain that $S\left(2 K_{1,3} \cup 3 K_{2}\right)=\emptyset$.

Corollary 5. If $S\left(2 K_{3} \cup 3 K_{2} \cup K_{1}\right)=\emptyset$ then $S\left(2 K_{3} \cup K_{1,3} \cup 2 K_{2}\right)$, $S\left(2 K_{3} \cup 4 K_{2}\right)$, $S\left(K_{3} \cup K_{1,4} \cup 3 K_{2}\right)=\emptyset$.

Proof. Let $F_{1}=2 K_{3} \cup 3 K_{2} \cup K_{1}$. Considering $F_{3}=2 K_{3} \cup 3 K_{2} \cup K_{1}$ we have that $S\left(2 K_{3} \cup 4 K_{2}\right)=\emptyset$. Considering $F_{3}=2 K_{3} \cup 2 K_{2} \cup 3 K_{1}$ we obtain that $S\left(2 K_{3} \cup K_{1,3} \cup 2 K_{2}\right)=$ $\emptyset$. Finally, considering $F_{3}=K_{3} \cup 3 K_{2} \cup 4 K_{1}$ we obtain that $S\left(K_{3} \cup K_{1,4} \cup 3 K_{2}\right)=\emptyset$.

Consequently, we have that if $S(F)=\emptyset$ for any $F \in \mathcal{F}=\left\{4 K_{3} \cup K_{2}, 3 K_{3} \cup K_{1,4}, 2 K_{3} \cup\right.$ $\left.2 K_{1,3}, K_{3} \cup 2 K_{2} \cup 5 K_{1}, 2 K_{3} \cup 3 K_{2} \cup K_{1}\right\}$ then $R\left(P_{4}, C_{4}, K_{4}\right)=14$.

Now, we are going to prove the main result of this subsection.

Theorem 6. $R\left(P_{4}, C_{4}, K_{4}\right)=14$. 
Proof. It is enough to prove that for any $F \in \mathcal{F}$ then $S(F)=\emptyset$.

There is a coloring in $S\left(5 K_{1}\right)$ for every $\left(C_{4}, K_{4} ; 5\right)$-coloring, thus $\left|S\left(5 K_{1}\right)\right|=13$. From this set, applying Algorithm 1, we obtain the sets $S\left(K_{2} \cup 4 K_{1}\right), S\left(2 K_{2} \cup 3 K_{1}\right), S\left(3 K_{2} \cup\right.$ $\left.2 K_{1}\right), S\left(4 K_{2} \cup K_{1}\right), S\left(5 K_{2}\right), S\left(K_{3} \cup 4 K_{2}\right), S\left(2 K_{3} \cup 3 K_{2}\right)$, the cardinalities of which are 122, 1012, 4808, 8569, 2676, 7466 and 968. From $S\left(2 K_{3} \cup 3 K_{2}\right)$, applying Algorithm 2, we generate the sets $S\left(2 K_{3} \cup 2 K_{2} \cup 2 K_{1}\right), S\left(2 K_{3} \cup K_{2} \cup 4 K_{1}\right)$ and $S\left(2 K_{3} \cup 6 K_{1}\right)$, the cardinalities of which are 944, 84 and 1. From $S\left(2 K_{3} \cup 6 K_{1}\right)$, applying Algorithm 1, we obtain the sets $S\left(2 K_{3} \cup K_{1,3} \cup 3 K_{1}\right)$, and $S\left(2 K_{3} \cup 2 K_{1,3}\right)$, the cardinalities of which are 5 and 0 , respectively. Thus $S\left(2 K_{3} \cup 2 K_{1,3}\right)=\emptyset$.

From $S\left(2 K_{3} \cup K_{2} \cup 4 K_{1}\right)$, applying Algorithm 2, we obtain that $S\left(K_{3} \cup 2 K_{2} \cup 5 K_{1}\right)=\emptyset$.

From $S\left(2 K_{3} \cup 3 K_{2}\right)$, applying Algorithm 1, we generate the set $S\left(3 K_{3} \cup 2 K_{2}\right)$. Its cardinality is 1. From $S\left(3 K_{3} \cup 2 K_{2}\right)$, applying Algorithm 2, we obtain $S\left(3 K_{3} \cup K_{2} \cup 2 K_{1}\right)$ and $S\left(3 K_{3} \cup 4 K_{1}\right)$. Their cardinalities are 2 and 1 . From $S\left(3 K_{3} \cup 4 K_{1}\right)$, applying Algorithm 1 , we have that $S\left(3 K_{3} \cup K_{1,4}\right)=\emptyset$.

From $S\left(3 K_{3} \cup 2 K_{2}\right)$, applying Algorithm 1, we obtain that $S\left(4 K_{3} \cup K_{2}\right)=\emptyset$ and, finally, from $S\left(3 K_{3} \cup 2 K_{2}\right)$, applying Algorithm 2, we have that $S\left(2 K_{3} \cup 3 K_{2} \cup K_{1}\right)=\emptyset$.

\section{$3.2 R\left(C_{4}, H_{1}, H_{2}\right)$}

To generate subfamilies of $\left(C_{4}, H_{1}, H_{2} ; n\right)$, where $H_{1}, H_{2} \in\left\{K_{3}, K_{4}-e\right\}$ we used the following algorithm.

\section{Algorithm 7. Extension}

Input: coloring $G \in\left(C_{4}, H_{1}, H_{2} ; n\right)$

Output: set of all one-vertex extensions of $G$ which belong to $\left(C_{4}, H_{1}, H_{2} ; n+1\right)$

For $R\left(C_{4}, K_{4}-e, K_{3}\right)$ we used the next algorithm. Let $H^{-}=K_{2}$ if $H=K_{3}$, and $H^{-}=P_{3}$ if $H=K_{4}-e$.

Algorithm 8. Merge

Input: coloring $G_{1} \in\left(C_{4}, H, P_{3} ; n\right)$ and $G_{2} \in\left(C_{4}, H^{-}, K_{4}-e ; m\right)$

Output: set of all colorings $G \in\left(C_{4}, H, K_{4}-e ; n+m+1\right)$ such that $G_{1}=G\left[N_{2}(v)\right]$ and $G_{2}=G\left[N_{3}(v)\right]$

Algorithm 7 is a standard procedure in graph theoretical computations. In case of generated subfamilies of $\left(C_{4}, H_{1}, H_{2} ; n\right)$, where $H_{1}, H_{2} \in\left\{K_{3}, K_{4}-e\right\}$ we cannot use it alone because we would have to keep collections of nonisomorphic colorings which are to large. Algorithm 7 is used to determine the collections of colorings $\left(C_{4}, P_{3}, K_{3} ; n\right)$ for $n \leqslant 7,\left(C_{4}, K_{4}-e, K_{2} ; n\right)$ for $n \leqslant 6$ and $\left(C_{4}, P_{3}, K_{4}-e ; n\right)$ for $n \leqslant 8$. The colorings from these collections were used as the parameters of Algorithm 8. Both of these algorithms are often used to determine Ramsey numbers (see $[2,25]$ ) therefore we do not discuss them in detail.

Let $t(n)$ denote the maximum number of edges of a graph with $n$ vertices not containing a $C_{4}$ as a subgraph.

Theorem 9. $R\left(C_{4}, K_{4}-e, K_{3}\right)=17$. 
Proof. Since $R\left(C_{4}, K_{4}-e, K_{3}\right) \geqslant R\left(C_{4}, K_{3}, K_{3}\right)=17$ [11], we obtain the lower bound. To obtain the upper bound we use the following computations. Since $R\left(C_{4}, P_{3}, K_{3}\right)=8$ and $R\left(C_{4}, K_{4}-e, K_{2}\right)=7[1]$, then for every vertex $u$ we have $d_{2}(u) \leqslant 7$ and $d_{3}(u) \leqslant 6$. Since $t(17)=36$ [8], then every coloring of $\left(C_{4}, K_{4}-e, K_{3} ; 17\right)$ must contain a vertex $v$ such that $d_{1}(v) \leqslant 4$. There are only 3 possibilities:

- There exists a vertex $v$ such that $d_{1}(v)=3, d_{2}(v)=7$ and $d_{3}(v)=6$.

We use Algorithm 8 for every graph $G_{1} \in\left(C_{4}, P_{3}, K_{3} ; 7\right)$ and $G_{2} \in\left(C_{4}, K_{4}-e, K_{2} ; 6\right)$ and find 8 colorings of $\left(C_{4}, K_{4}-e, K_{3} ; 14\right)$. Next we use Algorithm 7 to one-vertex extensions of these 8 colorings and obtain subfamilies of $\left(C_{4}, K_{4}-e, K_{3} ; n\right)$ for $n \in\{15,16,17\}$. Cardinalities of these sets are $6,43,0$, respectively.

- There exists a vertex $v$ such that $d_{1}(v)=4, d_{2}(v)=7$ and $d_{3}(v)=5$.

Similarly, for every $G_{1} \in\left(C_{4}, P_{3}, K_{3} ; 7\right)$ and $G_{2} \in\left(C_{4}, K_{4}-e, K_{2} ; 5\right)$ we found 26355 colorings of $\left(C_{4}, K_{4}-e, K_{3} ; 13\right)$. Next, we computed subsets of $\left(C_{4}, K_{4}-e, K_{3} ; n\right)$ for $n \in\{14,15,16,17\}$, the cardinalities of which are 470854, 515882, 3444, 0 , respectively.

- There exists a vertex $v$ such that $d_{1}(v)=4, d_{2}(v)=6$ and $d_{3}(v)=6$.

Again, for every $G_{1} \in\left(C_{4}, P_{3}, K_{3} ; 6\right)$ and $G_{2} \in\left(C_{4}, K_{4}-e, K_{2} ; 6\right)$ we found 132266 colorings of $\left(C_{4}, K_{4}-e, K_{3} ; 13\right)$. Next, we computed subsets of $\left(C_{4}, K_{4}-e, K_{3} ; n\right)$ for $n \in\{14,15,16,17\}$, the cardinalities of which are $4077662,8109281,56653,0$, respectively.

Finally, this means that $\left(C_{4}, K_{4}-e, K_{3} ; 17\right)=\emptyset$ and $R\left(C_{4}, K_{4}-e, K_{3}\right)=17$.

In order to prove some Theorems 11 and 13, we use the following lemma:

Lemma 10. Let $F$ be a graph of order $n$ and let $v_{1}, v_{2}, v_{3} \in V(F)$, such that $v_{i}$, for $i=1,2,3$, is adjacent to at least $\left\lfloor\frac{n}{3}\right\rfloor+1$ vertices of $V(F) \backslash\left\{v_{1}, v_{2}, v_{3}\right\}$. Then $C_{4}$ is a subgraph of $G$.

Proof. Let $a_{i}$ be the number of vertices of $V(F) \backslash\left\{v_{1}, v_{2}, v_{3}\right\}$ adjacent to $v_{i}$ and nonadjacent to the other two vertices of $\left\{v_{1}, v_{2}, v_{3}\right\}$, let $b_{i, j}$ be the number of vertices of $V(F) \backslash\left\{v_{1}, v_{2}, v_{3}\right\}$ adjacent to $v_{i}$ and $v_{j}$ and non-adjacent to the other one vertex of $\left\{v_{1}, v_{2}, v_{3}\right\}$ and let $c_{1,2,3}$ be the number of vertices of $V(F) \backslash\left\{v_{1}, v_{2}, v_{3}\right\}$ adjacent to $v_{1}$, $v_{2}$ and $v_{3}$. Then $a_{1}+a_{2}+a_{3}+b_{1,2}+b_{1,3}+b_{2,3}+c_{1,2,3} \leqslant n-3$.

Since $v_{i}$ is adjacent at least to $\left\lfloor\frac{n}{3}\right\rfloor+1$ vertices of $V(G) \backslash\left\{v_{1}, v_{2}, v_{3}\right\}$, we have that $a_{1}+b_{1,2}+b_{1,3}+c_{1,2,3} \geqslant\left\lfloor\frac{n}{3}\right\rfloor+1, a_{2}+b_{1,2}+b_{2,3}+c_{1,2,3} \geqslant\left\lfloor\frac{n}{3}\right\rfloor+1$ and $a_{3}+b_{1,3}+b_{2,3}+c_{1,2,3} \geqslant$ $\left\lfloor\frac{n}{3}\right\rfloor+1$.

Consequently, $a_{1}+a_{2}+a_{3}+2 b_{1,2}+2 b_{1,3}+2 b_{2,3}+3 c_{1,2,3} \geqslant 3\left\lfloor\frac{n}{3}\right\rfloor+3 \geqslant n+1$ and $b_{1,2}+b_{1,3}+b_{2,3}+2 c_{1,2,3} \geqslant 4$. Then there are $i$ and $j$ such that $b_{i, j}+c_{1,2,3} \geqslant 2, v_{i}$ and $v_{j}$ have at least two common neighbors belonging to $V(F) \backslash\left\{v_{1}, v_{2}, v_{3}\right\}$ and $C_{4}$ is a subgraph of $F$. 
We prove that adding an edge to $K_{3}$ leaves its Ramsey number unchanged, such as in the following theorem.

Theorem 11. $R\left(C_{4}, K_{4}-e, K_{3}+e\right)=R\left(C_{4}, K_{4}-e, K_{3}\right)=17$.

Proof. By Theorem 9 and by the monotonicity of Ramsey numbers we have that $17=R\left(C_{4}, K_{4}-e, K_{3}\right) \leqslant R\left(C_{4}, K_{4}-e, K_{3}+e\right)$. Assume, towards a contradiction, that $R\left(C_{4}, K_{4}-e, K_{3}\right)<R\left(C_{4}, K_{4}-e, K_{3}+e\right)$. Let $G$ be a $\left(C_{4}, K_{4}-e, K_{3}+e ; 17\right)$ coloring. There is a green triangle in $G$. Let $\left\{v_{1}, v_{2}, v_{3}\right\}$ be the vertices of a green triangle of $G$. Since $R\left(C_{4}, P_{3}, K_{3}+e\right)=8[1]$, then $\left|N_{2}\left(v_{i}\right)\right| \leqslant 7$ and $\left|N_{1}\left(v_{i}\right)\right| \geqslant 7$ for $i \in\{1,2,3\}$. By Lemma 10, we obtain a red $C_{4}$, a contradiction.

Theorem 12. $R\left(C_{4}, K_{4}-e, K_{4}-e\right)=19$.

Proof. Lower bound $R\left(C_{4}, K_{4}-e, K_{4}-e\right) \geqslant 19$ is presented in [9]. To obtain the upper bound we use similar computations as in the proof of Theorem 9 . Since $R\left(C_{4}, P_{3}, K_{4}-e\right)=$ $R\left(C_{4}, K_{4}-e, P_{3}\right)=9$ [1], then for every vertex $u$ we have $d_{2}(u) \leqslant 8$ and $d_{3}(u) \leqslant 8$. Since $t(19)=42[8]$, then every coloring of $\left(C_{4}, K_{4}-e, K_{3} ; 19\right)$ must contain vertex $v$ such that $d_{1}(v) \leqslant 4$. There are only 4 possibilities:

- There is a vertex $v$ such that $d_{1}(v)=4, d_{2}(v)=7$ and $d_{3}(v)=7$.

We use Algorithm 8 for every graph $G_{1} \in\left(C_{4}, P_{3}, K_{4}-e ; 7\right)$ and $G_{2} \in\left(C_{4}, K_{4}-\right.$ $\left.e, P_{3} ; 7\right)$ and find 621308 colorings of $\left(C_{4}, K_{4}-e, K_{4}-e ; 15\right)$. Next, we use Algorithm 7 to one-vertex extensions of these colorings and obtain subfamilies of $\left(C_{4}, K_{4}-e, K_{3} ; n\right)$ for $n \in\{16,17,18,19\}$. Cardinalities of these sets are 731002 , $18285,7,0$, respectively.

- There is a vertex $v$ such that $d_{1}(v)=4, d_{2}(v)=8$ and $d_{3}(v)=6$, (a case in which $d_{2}(v)=6$ and $d_{3}(v)=8$ is symmetrical).

Similarly, for every $G_{1} \in\left(C_{4}, P_{3}, K_{4}-e ; 8\right)$ and $G_{2} \in\left(C_{4}, K_{4}-e, P_{3} ; 6\right)$ we found 10488 colorings of $\left(C_{4}, K_{4}-e, K_{4}-e ; 15\right)$. Next, we computed subsets of $\left(C_{4}, K_{4}-\right.$ $\left.e, K_{4}-e ; n\right)$ for $n \in\{16,17,18\}$, the cardinalities of which are 28733, 1807, 0 , respectively.

- There is a vertex $v$ such that $d_{1}(v)=3, d_{2}(v)=8$ and $d_{3}(v)=7$, (a case in which $d_{2}(v)=7$ and $d_{3}(v)=8$ is symmetrical).

In this case Algorithm 8 returns an empty set of colorings.

- There is a vertex $v$ such that $d_{1}(v)=2, d_{2}(v)=8$ and $d_{3}(v)=8$.

In this case Algorithm 8 returns an empty set of colorings.

We state that set $\left(C_{4}, K_{4}-e, K_{4}-e ; 19\right)=\emptyset$ and $R\left(C_{4}, K_{4}-e, K_{4}-e\right)=19$.

Also, we have:

Theorem 13. $R\left(C_{4}, K_{3}+e, K_{4}\right) \leqslant \max \left\{R\left(C_{4}, K_{3}, K_{4}\right), 29\right\} \leqslant 32$. 
Proof. We know that $27 \leqslant R\left(C_{4}, K_{3}, K_{4}\right) \leqslant 32[9,28]$. Let us suppose that there exists $G$ a $\left(C_{4}, K_{3}+e, K_{4} ; \max \left\{R\left(C_{4}, K_{3}, K_{4}\right), 29\right\}\right)$-coloring. There is a blue triangle in $G$. Let $v_{1}$, $v_{2}, v_{3}$ be the vertices of a blue triangle. To avoid a blue $K_{4}+e$ we have that $d_{2}\left(v_{i}\right)=2$ and, since $R\left(C_{4}, K_{3}+e, K_{3}\right)=17, d_{3}\left(v_{i}\right) \leqslant 16$. Thus $d_{1}\left(v_{i}\right) \geqslant \max \left\{R\left(C_{4}, K_{3}, K_{4}\right), 29\right\}-19 \geqslant$ $\left\lfloor\frac{\max \left\{R\left(C_{4}, K_{3}, K_{4}\right), 29\right\}}{3}\right\rfloor+1$. By Lemma 10 , we have a red $C_{4}$, a contradiction.

Theorem 14. $28 \leqslant R\left(C_{4}, K_{4}-e, K_{4}\right) \leqslant 36$.

Proof. To prove the lower bound, we present a $\left(C_{4}, K_{4}-e, K_{4} ; 27\right)$-coloring in the Appendix. Let us suppose that there exits $G$, a $\left(C_{4}, K_{4}-e, K_{4} ; 36\right)$-coloring. Since $R\left(C_{4}, P_{3}\right.$, $\left.K_{4}\right)=13$ and $R\left(C_{4}, K_{4}-e, K_{3}\right)=17$ then $\Delta_{2}(G) \leqslant 12, \Delta_{3}(G) \leqslant 16, \delta_{1}(G) \geqslant 7$, and $\left|E\left(G^{1}\right)\right| \geqslant 126$. Since $t(36) \leqslant 115[8]$, there is a contradiction.

\section{3 $R\left(K_{3}, H_{1}, H_{2}\right)$ and $R\left(K_{3}+e, H_{1}, H_{2}\right)$}

Theorem 15. $R\left(K_{3}+e, K_{4}-e, K_{4}-e\right)=R\left(K_{3}, K_{4}-e, K_{4}-e\right)$.

Proof. $R\left(K_{3}, K_{4}-e, K_{4}-e\right) \geqslant 21$ [27]. Let us suppose that $R\left(K_{3}+e, K_{4}-e, K_{4}-e\right)>$ $R\left(K_{3}, K_{4}-e, K_{4}-e\right)$, thus there exists $G$ a $\left(K_{3}+e, K_{4}-e, K_{4}-e ; R\left(K_{3}, K_{4}-e, K_{4}-e\right)\right)$ coloring and there is a red triangle in $G$. Let $v_{1}, v_{2}$ and $v_{3}$ be the vertices of a red triangle of $G$. We may also assume that $d_{2}\left(v_{1}\right) \geqslant 9$. Clearly $G\left[N_{2}\left(v_{1}\right)\right]$ contains no blue $P_{3}$. Assume that $w_{i}, 1 \leqslant i \leqslant 9$ are the 9 first vertices of $G\left[N_{2}\left(v_{1}\right)\right]$. Next, we consider the cases with respect to the number of blue edges in $G\left[N_{2}\left(v_{1}\right)\right]$ as follows.

- $G\left[N_{2}\left(v_{1}\right)\right]$ has 4 disjoint blue edges.

Assume that $\left\{w_{i}, w_{i+1}\right\}$ are blue for all $i \in\{1,3,5,7\}$. To avoid a blue $K_{4}-e$, without the loss of generality we can assume that $\left\{v_{2}, w_{i}\right\}$ are green for all $i \in$ $\{2,4,6,8\}$. Without the loss of generality we can assume that $\left\{w_{2}, w_{4}\right\},\left\{w_{6}, w_{8}\right\}$ are green, then $\left\{w_{2}, w_{6}\right\},\left\{w_{2}, w_{8}\right\},\left\{w_{4}, w_{6}\right\},\left\{w_{4}, w_{8}\right\}$ must be red. To prevent $\left\{v_{2}, w_{2}, w_{4}, w_{9}\right\}$ from forming a green $K_{4}-e$, for at least one $i \in\{2,4\}$, the edge $\left\{w_{i}, w_{9}\right\}$ is red. We have that $\left\{w_{6}, w_{9}\right\},\left\{w_{8}, w_{9}\right\}$ are green, then $\left\{v_{2}, w_{6}, w_{8}, w_{9}\right\}$ forms a green $K_{4}-e$.

- $G\left[N_{2}\left(v_{1}\right)\right]$ has 3 disjoint blue edges.

Clearly $G\left[N_{2}\left(v_{1}\right)\right]$ contains a $K_{7}-e$ with only red and green edges. By Lemma 2 in [27], we have either a red $K_{3}+e$ or a green $K_{4}-e$.

- $G\left[N_{2}\left(v_{1}\right)\right]$ has at most 2 disjoint blue edges.

Since $R\left(K_{3}+e, K_{4}-e\right)=7$ [7], we immediately obtain a red $K_{3}+e$ or a green $K_{4}-e$.

By using similar methods we obtain the following:

Theorem 16. $R\left(K_{3}, K_{4}-e, K_{4}\right) \leqslant \max \left\{R\left(K_{3}+e, K_{4}-e, K_{4}\right), 33\right\} \leqslant 41$. 
Proof. Let us suppose that $R\left(K_{3}+e, K_{4}-e, K_{4}\right)>\max \left\{R\left(K_{3}+e, K_{4}-e, K_{4}\right), 33\right\}$, thus there exists $G$ be a $\left(K_{3}+e, K_{4}-e, K_{4} ; \max \left\{R\left(K_{3}+e, K_{4}-e, K_{4}\right), 33\right\}\right)$-coloring and there is a red triangle in $G$. Let $v_{1}, v_{2}$ and $v_{3}$ be the vertices of a red triangle of $G$. We may also assume that $d_{2}\left(v_{1}\right) \geqslant 14$. Clearly $G\left[N_{2}\left(v_{1}\right)\right]$ does not contain any blue $P_{3}$. Assume that $w_{1}, w_{2}, \ldots, w_{14}$ are the 14 first vertices of $G\left[N_{2}\left(v_{1}\right)\right]$. Next, we consider the cases with respect to the number of blue edges in $G\left[N_{2}\left(v_{1}\right)\right]$ as follows.

- $G\left[N_{2}\left(v_{1}\right)\right]$ has 7 disjoint blue edges.

Assume that $\left\{w_{i}, w_{i+1}\right\}$ are blue for odd $1 \leqslant i \leqslant 13$. To avoid a blue $K_{4}-e$, we can assume that $\left\{v_{2}, w_{i}\right\}$ are green for even $2 \leqslant i \leqslant 14$. Since $R\left(K_{3}+e, K_{3}\right)=7$ [7], we immediately obtain a red $K_{3}+e$ or a green $K_{4}$.

- $G\left[N_{2}\left(v_{1}\right)\right]$ has 6 disjoint blue edges.

Assume that $\left\{w_{i}, w_{i+1}\right\}$ are blue for odd $1 \leqslant i \leqslant 11$. To avoid a blue $K_{4}-e$, we can assume that $\left\{v_{2}, w_{i}\right\}$ are green for even $2 \leqslant i \leqslant 12$. If $\left\{v_{2}, w_{13}\right\}$ is green, then by $R\left(K_{3}+e, K_{3}\right)=7$ [7], we have either a red $K_{3}+e$ or a green $K_{4}$. This means that $\left\{v_{2}, w_{13}\right\}$ is blue. Since $R\left(K_{3}, K_{3}\right)=6$, then we can assume there are two red triangles, let their vertices be $\left\{w_{2}, w_{4}, w_{6}\right\}$ and $\left\{w_{8}, w_{10}, w_{12}\right\}$. The remaining edges of a subgraph on $\left\{w_{2}, w_{4}, w_{6}, w_{8}, w_{10}, w_{12}\right\}$ are green. There is at least one green edge in the subgraph on vertices $\left\{w_{1}, w_{3}, w_{5}, w_{7}, w_{9}, w_{11}\right\}$, say $\left\{w_{1}, w_{3}\right\}$, then $\left\{w_{1}, w_{3}, w_{6}, w_{8}\right\}$ forms a green $K_{4}$.

- $G\left[N_{2}\left(v_{1}\right)\right]$ has 5 disjoint blue edges.

Assume that $\left\{w_{i}, w_{i+1}\right\}$ are blue for all $i \in\{1,3,5,7,9\}$. Let us consider a subgraph $H$ on vertices $\left\{w_{2}, w_{4}, w_{6}, w_{8}, w_{10}, w_{11}, w_{12}, w_{13}, w_{14}\right\}$. Since $R\left(K_{3}, K_{4}\right)=9$, then $H$ contain a red triangle. To avoid a red $K_{3}+e$ and a green $K_{4}$ the remaining vertices of $H$ contain a next red triangle. By the fact that a subgraph on vertices $\left\{w_{1}, w_{3}, w_{5}, w_{7}, w_{9}\right\}$ has at least one green edge, we immediately obtain a green $K_{4}$.

- $G\left[N_{2}\left(v_{1}\right)\right]$ has at most 4 disjoint blue edges.

Since $R\left(K_{3}+e, K_{4}\right)=10$ [7], we immediately obtain a red $K_{3}+e$ or a green $K_{4} \cdot \square$

In order to prove Theorems 20 and 21 we use some definitions and lemmas.

Lemma 17. Let $G$ be a $\left(K_{3}, K_{4}-e, K_{4} ; 41\right)$-coloring. For any $v \in V(G), G^{2}\left[N_{2}(v)\right] \in$ $\left\{7 K_{2}, 6 K_{1} \cup 2 K_{1}\right\}$.

Proof. Let $v_{0} \in V(G)$ such that $d_{2}\left(v_{0}\right)=\Delta_{2}(G)$. Then $d_{2}\left(v_{0}\right) \leqslant R\left(K_{3}, P_{3}, K_{4}\right)-1=16$. If $d_{2}\left(v_{0}\right)=16$, as $G\left[N_{2}\left(v_{0}\right)\right]$ is a $\left(K_{3}, P_{3}, K_{4} ; 16\right)$-coloring and $R\left(K_{3}, K_{4}\right)=9$ [12], we have that $G^{2}\left[N_{2}\left(v_{0}\right)\right]=8 K_{2}$. Let $x_{1}, \ldots, x_{8}$ be the edges of $G^{2}\left[N_{2}\left(v_{0}\right)\right]$ and let $w$ be one of the 24 vertices of $N_{1}\left(v_{0}\right) \cup N_{3}\left(v_{0}\right)$. To prevent a blue $K_{4}-e$, for at least one vertex $w_{i}$ of $x_{i}$, with $1 \leqslant i \leqslant 8$, the edge $\left\{w, w_{i}\right\}$ is red or green. Then $G\left[\left\{w, w_{1}, \ldots, w_{8}\right\}\right]$ has not blue edges and there is a red $K_{3}$ or a green $K_{4}$. There is a contradiction, thus $\Delta_{2}(G) \leqslant 15$. 
If $d_{2}\left(v_{0}\right)=15$, as $G\left[N_{2}\left(v_{0}\right)\right]$ is a $\left(K_{3}, P_{3}, K_{4} ; 15\right)$-coloring, then $G^{2}\left[N_{2}\left(v_{0}\right)\right]=7 K_{2} \cup K_{1}$. Let $x_{1}, \ldots, x_{7}$ be the edges of $G^{2}\left[N_{2}\left(v_{0}\right)\right]$, let $u$ be the isolated vertex of $G^{2}\left[N_{2}\left(v_{0}\right)\right]$ and let $w$ be some of the at least 11 vertices of $\left(N_{1}\left(v_{0}\right) \cup N_{3}\left(v_{0}\right)\right) \backslash N_{2}(u)$. To prevent a blue $K_{4}-e$, for at least one vertex $w_{i}$ of $x_{i}$, with $1 \leqslant i \leqslant 7$, the edge $\left\{w, w_{i}\right\}$ is red or green. Then $G\left[\left\{w, u, w_{1}, \ldots, w_{7}\right\}\right]$ has not blue edges and there is a red $K_{3}$ or a green $K_{4}$. Thus $\Delta_{2}(G) \leqslant 14$.

Let $v \in V(G)$. Since $d_{1}(v) \leqslant R\left(K_{2}, K_{4}-e, K_{4}\right)-1=10, d_{2}(v) \leqslant 14, d_{3}(v) \leqslant$ $R\left(K_{3}, K_{4}-e, K_{3}\right)-1=16$ and $d_{1}(v)+d_{2}(v)+d_{3}(v)=40$, we have that $d_{1}(v)=$ $10, d_{2}(v)=14, d_{3}(v)=16$ and, as $G\left[N_{2}(v)\right]$ is a $\left(K_{3}, P_{3}, K_{4} ; 14\right)$-coloring, $G^{2}\left[N_{2}(v)\right] \in$ $\left\{7 K_{2}, 6 K_{2} \cup 2 K_{1}\right\}$.

Let $A=\left\{v \in V(G): N_{2}(v)=6 K_{2} \cup 2 K_{1}\right\}$. We have:

Lemma 18. $A \neq \emptyset$.

Proof. If $A=\emptyset$ then every vertex belongs to 7 blue triangles and $G$ has $\frac{41 \cdot 7}{3}$ blue triangles. This number is not an integer and there is a contradiction.

For any $v \in A$ we define $D_{v}=\left\{u \in N_{2}(v): d_{G^{2}\left[N_{2}(v)\right]}(u)=1\right\}$. Clearly $\left|N_{2}(v) \backslash D_{v}\right|=2$ and $N_{2}(v) \backslash D_{v} \subseteq A$.

Lemma 19. Let $v_{1} \in A$ and $v_{0}, v_{2} \in N_{2}\left(v_{1}\right) \backslash D_{v_{1}}$, then $N_{2}\left(v_{0}\right) \cap N_{2}\left(v_{2}\right)=\left\{v_{1}\right\}$.

Proof. If $\left|N_{2}\left(v_{0}\right) \cap N_{2}\left(v_{2}\right) \backslash\left\{v_{1}\right\}\right| \geqslant 1$, since $\left|N_{2}\left(v_{0}\right) \backslash\left\{v_{1}\right\}\right|,\left|N_{2}\left(v_{2}\right) \backslash\left\{v_{1}\right\}\right|=13$, $N_{2}\left(v_{0}\right) \backslash\left\{v_{1}\right\}, N_{2}\left(v_{2}\right) \backslash\left\{v_{1}\right\} \subset N_{1}\left(v_{1}\right) \cup N_{3}\left(v_{1}\right)$ and $\left|N_{1}\left(v_{1}\right) \cup N_{3}\left(v_{1}\right)\right|=26$, we have that there exists $w \in\left(N_{1}\left(v_{1}\right) \cup N_{3}\left(v_{1}\right)\right) \backslash\left(N_{2}\left(v_{0}\right) \cup N_{2}\left(v_{2}\right)\right)$. Let $x_{1}, \ldots, x_{6}$ be the edges of $G^{2}\left[N_{2}\left(v_{1}\right)\right]$. To prevent a blue $K_{4}-e$, for at least one vertex $w_{i}$ of $x_{i}$, with $1 \leqslant i \leqslant 6$, the edge $\left\{w, w_{i}\right\}$ is red or green. Then $G\left[\left\{w, v_{0}, v_{2}, w_{1}, \ldots, w_{6}\right\}\right]$ has not blue edges and, as $R\left(K_{3}, K_{4}\right)=9$, there is a red $K_{3}$ or a green $K_{4}$, a contradiction.

Now, we have:

Theorem 20. $R\left(K_{3}, K_{4}-e, K_{4}\right) \leqslant 41$.

Proof. Let $G$ be a $\left(K_{3}, K_{4}-e, K_{4} ; 41\right)$-coloring. Let $v_{1} \in A, v_{0}, v_{2} \in N_{2}\left(v_{1}\right) \backslash D_{v_{1}}$ and $v_{3} \in\left(N_{2}\left(v_{2}\right) \backslash D_{v_{2}}\right) \backslash\left\{v_{1}\right\}$. By Lemma 19, $N_{2}\left(v_{0}\right) \cap N_{2}\left(v_{2}\right)=\left\{v_{1}\right\}$ and $N_{2}\left(v_{1}\right) \cap N_{2}\left(v_{3}\right)=$ $\left\{v_{2}\right\}$. Let $B=V(G) \backslash\left\{v_{0}, v_{1}, v_{2}, v_{3}\right\} \backslash\left(D_{v_{1}} \cup D_{v_{2}}\right)$. As $|B|=13,\left|N_{2}\left(v_{0}\right) \backslash\left\{v_{1}, v_{3}\right\}\right|$, $\left|N_{2}\left(v_{3}\right) \backslash\left\{v_{0}, v_{2}\right\}\right| \geqslant 12$ and $N_{2}\left(v_{0}\right) \backslash\left\{v_{1}, v_{3}\right\}, N_{2}\left(v_{3}\right) \backslash\left\{v_{0}, v_{2}\right\} \subseteq B$, we have that $\left|\left(N_{2}\left(v_{0}\right) \backslash\left\{v_{1}, v_{3}\right\}\right) \cap\left(N_{2}\left(v_{3}\right) \backslash\left\{v_{0}, v_{2}\right\}\right)\right| \geqslant 11$ and, as $R\left(K_{3}, K_{4}\right)=9, G\left[\left(N_{2}\left(v_{0}\right) \backslash\left\{v_{1}, v_{3}\right\}\right) \cap\right.$ $\left.\left(N_{2}\left(v_{3}\right) \backslash\left\{v_{0}, v_{2}\right\}\right)\right]$ contains a blue edge and $G\left[\left(\left(N_{2}\left(v_{0}\right) \backslash\left\{v_{1}, v_{3}\right\}\right) \cap\left(N_{2}\left(v_{3}\right) \backslash\left\{v_{0}, v_{2}\right\}\right)\right) \cup\right.$ $\left.\left\{v_{0}, v_{3}\right\}\right]$ contains a blue $K_{4}-e$, a contradiction.

Theorem 21. $R\left(K_{3}+e, K_{4}, K_{4}\right) \leqslant \max \left\{R\left(K_{3}, K_{4}, K_{4}\right), 2 R\left(K_{3}, K_{3}, K_{4}\right)+2\right\} \leqslant 79$.

Proof. The result is obvious if $R\left(K_{3}+e, K_{4}, K_{4}\right)=R\left(K_{3}, K_{4}, K_{4}\right) \leqslant 79$. Assume that $R\left(K_{3}+e, K_{4}, K_{4}\right)>R\left(K_{3}, K_{4}, K_{4}\right)$, and let $G$ be a $\left(K_{3}+e, K_{4}, K_{4} ; R\left(K_{3}+e, K_{4}, K_{4}\right)-1\right)$ coloring. As $R\left(K_{3}+e, K_{4}, K_{4}\right)-1 \geqslant R\left(K_{3}, K_{3}, K_{4}\right)$, there are at least a triangle in $G$. Let $v$ be a vertex of a red triangle. To avoid a red $K_{3}+e, d_{1}(v)=2$, and $d_{2}(v), d_{3}(v) \leqslant R\left(K_{3}+\right.$ $\left.e, K_{3}, K_{4}\right)-1=R\left(K_{3}, K_{3}, K_{4}\right)-1$. As $R\left(K_{3}+e, K_{4}, K_{4}\right)-1=1+d_{1}(v)+d_{2}(v)+d_{3}(v)$, we have that $R\left(K_{3}+e, K_{4}, K_{4}\right) \leqslant 2 R\left(K_{3}, K_{3}, K_{4}\right)+2 \leqslant 64$. 


\section{4 $R\left(K_{4}-e, H_{1}, H_{2}\right)$}

In this subsection we show new upper bounds on $R\left(K_{4}-e, K_{4}-e, K_{4}\right)$ and $R\left(K_{3}, K_{4}, K_{4}\right)$. In order to obtain the main results of this subsection we use the following lemma, which has a computational proof.

Lemma 22. Every $\left(P_{3}, K_{4}-e, K_{4} ; 16\right)$-coloring has 8 red edges.

Theorem 23. $R\left(K_{4}-e, K_{4}-e, K_{4}\right) \leqslant 59$.

Proof. Let $G$ be a $\left(K_{4}-e, K_{4}-e, K_{4} ; 59\right)$-coloring and let $v \in V(G)$. It is straightforward that $d_{1}(v), d_{2}(v) \leqslant R\left(P_{3}, K_{4}-e, K_{4}\right)-1=16$ and $d_{3}(v) \leqslant R\left(K_{4}-e, K_{4}-e, K_{3}\right)-1 \leqslant 26$. Since $d_{1}(v)+d_{2}(v)+d_{3}(v)=58$, we have $d_{1}(v)=16$ and by Lemma 22, $G\left[N_{1}(v)\right]$ has 8 red edges, $v$ belongs to 8 red triangles and $G$ has $\frac{59.8}{3}$ red triangles. This number is not an integer and we have a contradiction.

In order to prove the last theorem we need the following lemma:

Lemma 24. Let $G$ be a $\left(K_{4}-e, K_{4}, K_{4} ; 113\right)$-coloring and let $v \in V(G)$. Then $d_{1}(v)=32$, $d_{2}(v), d_{3}(v)=40$ and $G^{1}\left[N_{1}(v)\right]=16 K_{2}$.

Proof. The proof of $\Delta_{1}(G) \leqslant 32$ is similar to the proof of Lemma 17 .

Since $d_{1}(v) \leqslant 32, d_{2}(v), d_{3}(v) \leqslant R\left(K_{4}-e, K_{3}, K_{4}\right)-1 \leqslant 40$ and $d_{1}(v)+d_{2}(v)+d_{3}(v)=$ 112 , we have that $d_{1}(v)=32, d_{2}(v)=d_{3}(v)=40$ and, as $G\left[N_{1}(v)\right]$ is a $\left(P_{3}, K_{4}, K_{4} ; 32\right)$ coloring and $R\left(K_{4}, K_{4}\right)=18$ [12], we have that $G^{1}\left[N_{1}(v)\right] \in\left\{16 K_{2}, 15 K_{2} \cup 2 K_{1}\right\}$.

Let us suppose $G^{1}\left[N_{1}(v)\right]=15 K_{2} \cup 2 K_{1}$. Let $x_{1}, \ldots, x_{15}$ be the edges of $G^{1}\left[N_{1}(v)\right]$, let $u_{1}$ and $u_{2}$ be the isolated vertices of $G^{1}\left[N_{1}(v)\right]$ and let $w$ be some of the at least 18 vertices of $\left(N_{2}(v) \cup N_{3}(v)\right) \backslash\left(N_{1}\left(u_{1}\right) \cup N_{1}\left(u_{2}\right)\right)$. To prevent a red $K_{4}-e$, for at least one vertex $w_{i}$ of $x_{i}$, with $1 \leqslant i \leqslant 15$, the edge $\left\{w, w_{i}\right\}$ is blue or green. Then $G\left[\left\{w, u_{1}, u_{2}, w_{1}, \ldots, w_{15}\right\}\right]$ has not red edges and, as $R\left(K_{4}, K_{4}\right)=18$ [12], there is a blue $K_{4}$ or a green $K_{4}$, thus there is a contradiction.

Theorem 25. $R\left(K_{4}-e, K_{4}, K_{4}\right) \leqslant 113$.

Proof. Let $G$ be a $\left(K_{4}-e, K_{4}, K_{4} ; 113\right)$-coloring. By Lemma 24 , every vertex of $G$ belongs to 16 blue triangles, thus $G$ has $\frac{113 \cdot 16}{3}$ blue triangles. This number is not an integer and we have a contradiction.

\section{Open cases}

The still open cases are the values of $R\left(H_{1}, K_{4}-e, K_{4}-e\right)$, with $H_{1} \in\left\{K_{3}, K_{3}+e, K_{4}-\right.$ $\left.e, K_{4}\right\}$, and $R\left(H_{1}, H_{2}, K_{4}\right)$, with $H_{1}, H_{2} \in\left\{C_{4}, K_{3}, K_{3}+e, K_{4}-e, K_{4}\right\}$. In general, the difficulty of these open cases depends on the structure of the graphs and almost always grows with the number of edges of the graphs. On the one hand, we think that the following cases should be solved $R\left(C_{4}, C_{4}, K_{4}\right), R\left(C_{4}, K_{3}, K_{4}\right), R\left(K_{3}, K_{4}-e, K_{4}-e\right)$ or $R\left(K_{3}, K_{4}, K_{4}\right)$. On the other hand there is no progress in the bounds on $R\left(K_{4}, K_{4}, K_{4}\right)$ 
since 1982 [13] and the difference between its bounds are 108. Probably, this case can never be solved.

Also, we expect that the values of the open cases are closer to the lower bounds than the upper bounds and we conjecture that $R\left(K_{3}+e, H, K_{4}\right)=R\left(K_{3}, H, K_{4}\right)$ for $H \in\left\{C_{4}, K_{4}-e, K_{4}\right\}$.

Acknowledgement. We would like to thank the anonymous reviewers whose suggestions led to improved presentation of this work.

\section{References}

[1] J. Arste, K. Klamroth, and I. Mengersen. Three Color Ramsey Numbers for Small Graphs. Utilitas Mathematica, 49:85-96, 1996.

[2] A. Babak, S. P. Radziszowski and Kung-Kuen Tse. Computation of the Ramsey Number $R\left(B_{3}, K_{5}\right)$. Bulletin of the Institute of Combinatorics and Its Applications, 41:71-76, 2004.

[3] A. Bialostocki and J. Schönheim. On Some Turán and Ramsey Numbers for $C_{4}$. Graph Theory and Combinatorics, ed. B. Bollobás, Academic Press, London, 29-33, 1984.

[4] S. A. Burr and P. Erdös. Generalizations of a Ramsey-Theoretic Result of Chvátal. Journal of Graph Theory, 7:39-51, 1983.

[5] S. A. Burr and J. A. Roberts. On Ramsey Numbers for stars. Utilitas Mathematica, 4:217-220, 1973.

[6] V. Chvátal and F. Harary. Generalized Ramsey Theory for Graphs, II. Small Diagonal Numbers. Proceedings of the American Mathematical Society, 32:389-394, 1972.

[7] V. Chvátal and F. Harary. Generalized Ramsey Theory for Graphs, III. Small OffDiagonal Numbers. Pacific Journal of Mathematics, 41:335-345, 1972.

[8] C. Clapham, A. Flockhart, and J. Sheehan. Graphs Without Four-Cycles. Journal of Graph Theory, 13:29-47, 1989.

[9] J. Dybizbański and T. Dzido. On Some Ramsey Numbers for Quadrilaterals. Electronic Journal of Combinatorics, 18:\#P154, 12 pages, 2011. http://www. combinatorics.org/

[10] G. Exoo. On the Three Color Ramsey Number of $K_{4}-$ e. Discrete Mathematics, 89:301-305, 1991.

[11] G. Exoo and D. F. Reynolds. Ramsey Numbers Based on $C_{5}$-Decompositions. Discrete Mathematics, 71:119-127, 1988.

[12] R. E. Greenwood and A.M. Gleason. Combinatorial Relations and Chromatic Graphs. Canadian Journal of Mathematics, 7:1-7, 1955. 
[13] R. Hill and R.W. Irving. On Group Partitions Associated with Lower Bounds for Symmetric Ramsey Numbers. European Journal of Combinatorics, 3:35-50, 1982.

[14] R. W. Irving. Generalized Ramsey Numbers for Small Graphs. Discrete Mathematics, 9:251-264, 1974.

[15] M. S. Jacobson. On the Ramsey Number for Stars and a Complete Graph. Ars Combinatoria, 17:167-172, 1984.

[16] J. G. Kalbfleisch. Chromatic Graphs and Ramsey's Theorem Ph D. thesis, University of Waterloo, 1966.

[17] K. Klamroth and I. Mengersen. The Ramsey Number of $r\left(K_{1,3}, C_{4}, K_{4}\right)$. Utilitas Mathematica, 52:65-81, 1997.

[18] D. L. Kreher, L. Wei, and S. P. Radziszowski. Lower Bounds for Multi-Colored Ramsey Numbers From Group Orbits. Journal of Combinatorial Mathematics and Combinatorial Computing, 4:87-95, 1988.

[19] S. Wolfram. Mathematica 8.0, 2010. http://www.wolfram.com/mathematica/

[20] B.D. McKay. Practical Graph Isomorphism. Congressus Numerantium, 30:45-87, 1981.

[21] B.D. McKay. Nauty User's Guide (Version 2.4). Technical Report TR-CS-90-02, Department of Computer Science, Australian National University, 1990. http://cs . anu. edu. au/ bdm/nauty.

[22] K. Piwakowski. A New Upper Bound for $R_{3}\left(K_{4}-e\right)$. Congressus Numerantium, 128:135-141, 1997.

[23] K. Piwakowski and S.P. Radziszowski. $30 \leqslant R(3,3,4) \leqslant 31$. Journal of Combinatorial Mathematics and Combinatorial Computing, 27:135-141, 1998.

[24] S. P. Radziszowski. Small Ramsey numbers. The Electronic Journal of Combinatoric, Dynamic Survey 1:\#13, 2011. http://www.combinatorics.org/

[25] S. P. Radziszowski, J. Stinehour and Kung-Kuen Tse. Computation of the Ramsey Number $R\left(W_{5}, K_{5}\right)$. Bulletin of the Institute of Combinatorics and its Applications, 47:53-57, 2006.

[26] C. U. Schulte. Ramsey-Zahlen für Bäume und Kreise. Ph D. thesis, Heinrich-HeineUniversität Düsseldorf, 1992.

[27] D. S. Shetler, M. A. Wurtz, and S.P. Radziszowski. On Some Multicolor Ramsey Numbers Involving $K_{3}+e$ and $K_{4}-e$. To appear in the SIAM Journal on Discrete Mathematics.

[28] Xiaodong Xu, Zehui Shao, and S. P. Radziszowski. Bounds on Some Ramsey Numbers Involving Quadrilateral. Ars Combinatoria, 90:337-344, 2009.

[29] Yang Yuansheng and P. Rowlinson. The Third Ramsey Numbers for Graphs with at Most Four Edges. Discrete Mathematics, 125:399-406, 1994. 


\title{
5 Appendix
}

\author{
X022122211212 \\ 0X22221202212 \\ 22X2111022222 \\ 222X221220120 \\ $1212 \mathrm{X} 12021222$ \\ 22121X0122222 \\ $211120 \mathrm{X} 22112$ \\ $2202012 X 12021$ \\ $10222221 \mathrm{X} 2211$ \\ $122012222 \mathrm{X} 120$ \\ $2221221021 \mathrm{X} 2$ \\ $11222212122 \mathrm{X} 2$ \\ $222022211012 \mathrm{X}$
}

Figure 1: Matrix of $\left(P_{4}, C_{4}, K_{4} ; 13\right)$-coloring.

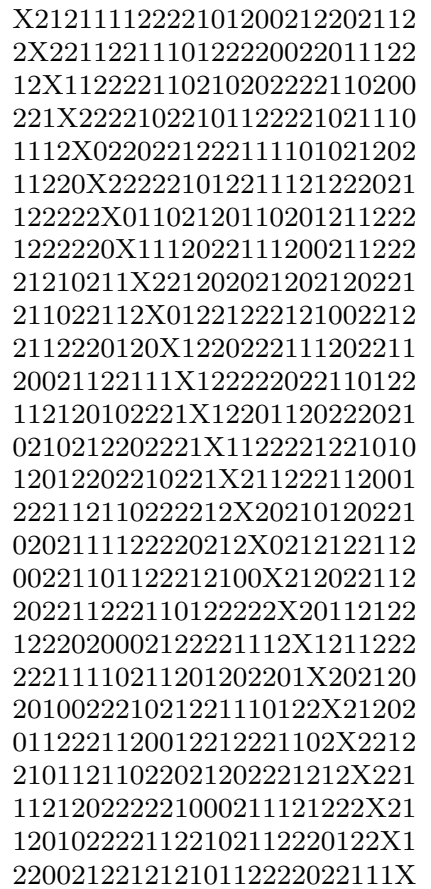

Figure 2: Matrix of $\left(C_{4}, K_{4}-e, K_{4} ; 27\right)$-coloring. 